\title{
Neptune's satellites predicted?
}

A theory believed to be successful in predicting the satellites of Uranus is not widely accepted. Much will hang on whether it has been successfully applied to Neptune.

Last week's encounter of Voyager 2 with Neptune will be, when the data have been analysed, a fateful occasion for Dr Andrew Prentice of Monash University in Melbourne. For the past several years, Prentice (a mathematician by trade) has had some success in predicting the properties of the outer planets and their satellites, most conspicuously at the encounter between Voyager 2 and Uranus in 1986. By definition, the encounter with Neptune will have been the last chance to test the accuracy of his prediction - there are no more planets left. But it is unlikely that the argument surrounding Prentice's work, which raises absorbing questions about the validity of predictive theories whose mechanisms are disputed, will be stilled as quickly.

Prentice himself makes no secret of the origin of his theory, which is based on the attempt by Laplace, in the eighteenth century, to relate the radii of the orbits of the planets to each other. Indeed, Laplace's theory was an important part of his great work Exposition du Système du Monde by which he is most widely remembered. For the period, the insight is quite remarkable. It is no shame to Laplace that he was unable to describe a mechanism whereby rings of gas and dust could condense into planets.

That is what Prentice reckons to have done, in the process reaching conclusions about the way in which the radii of the satellites of the multi-satellited planets beginning with Jupiter are related to each other. For the regular satellite systems such as those of Uranus, in which the satellites revolve in the same direction as the rotation of the planet, there is held to be a constant ratio between the radii of successive planets. This is the prediction that worked spectacularly well for Uranus. But Neptune is different in at least one respect: Triton, the largest of its satellites, travels in an orbit inclined to the ecliptic and to the plane of Neptune's equator, and in a direction opposite to that of the planet's own rotation. Few dispute Prentice's assumption that Triton was formed separately, but probably from the same material as Neptune (and, for that matter, Pluto). Part of his present interest is in the radii of the other pro-grade satellites of Neptune. Another is in the chemical composition (and thus the mean density) of objects such as Triton, as calculated from the supposed history of the condensing gas cloud.
The argument about Prentice's "modern laplacian theory" centres not on the predictions, but about the mechanisms it supposes to control condensation. The driving force both for the shedding of successive planetary rings and the formation of the separate satellite systems is supposed to be turbulence caused and then sustained by convection, presumably from the evolving primitive Sun. If there is such a mechanism, and if it can be sustained, there is no doubt that the consequences for a contracting solar nebula would be profound. In the outer regions of the cloud, for example, turbulent velocities would be supersonic, while turbulent stress would enormously augment the normal pressure of the gas. People's quarrel with Prentice hangs most simply on the circumstance that turbulence is a dissipative process whose effect would be to heat the gases involved in the turbulent motions (as might be expected for convection).

So what will people say if Prentice's predictions for the satellites of Neptune are shown to be as accurate as those for the satellites of Uranus in 1986? There will, of course, be two opinions. Prentice, his associates and his supporters will no doubt be confirmed in their opinion that they are on to something. Nobody would expect them to behave differently. But, equally, their critics will continue to insist that, in the absence of a plausible mechanism, the theory has no status.

Where will the truth lie? It is appropriate to recall that, in Laplace's time, strictly empirical theories were respectable.

Dulong and Petit, for example, contemporaries of Laplace, made sense of the specific heats of metals in a manner not made respectable until the arrival of the kinetic theory. Their rule, then a relationship between specific heat and atomic weight, should then, of course, have fallen into disrepute with the discovery that electrical conduction is mediated by electrons, only to be resurrected with the arrival of the quantum theory.

Then, at least, strictly empirical theories were both common and acceptable, as they still are in many fields - the various rules about species diversity with which ecologists are concerned are good examples.

So may it be that Prentice has arrived at an empirical rule for relating the properties of satellite systems to each other, and happens merely to have put forward an implausible mechanism that will eventually be refined? That, no doubt, is how the critics will be answered if the Neptune predictions are borne out by the observations of Voyager 2. And there will be some (but not Prentice, who does not acknowledge that his mechanism is faulty) who complain that too slavish a concern for mechanisms is the enemy of progress. That argument would have force if the issue were essentially practical, say the prediction of the superconducting properties of various ternary oxides (and, in the absence of an understanding of the mechanism of high-temperature superconductivity, empirical theories have enjoyed a new lease of life in that field during the past two years).

But there are two reasons why the licence does not apply to the prediction of planetary satellite systems, of which the simplest and more trivial is that there are only limited data to account for. With Voyager 2 now past Neptune, and with the prospect that it will be years or even decades before there is detailed information on other planetary systems, further prediction has no practical purpose. The situation is much like that in particle physics, where empirical arithmetical rules relating the masses of known particles to each other are forever popping up, but where the true objective can only be a surer understanding of their physical basis. In that connection, plausibility is essential.

The more telling reason why Prentice's critics will not fall silent is that there is already a great deal of successful planetary science entailing only plausible mechanisms. Indeed, it is one of the achievements of the past few decades that so much has been done to understand the way in which the Solar System formed from primaeval gas mixed with stellar debris. On that view, there was indeed a condensing nebula, the variations of chemical composition from one planet to another are intelligible in terms of the condensation temperatures of their constituents, but there must be many features of the Solar System that will be explained only historically, as consequences of past events still unknown. None of that is unrespectable, or a manifestation of orthodoxy conspiring to suppress the heterodox.

Even so, it will be interesting to see whether Prentice's predictions for Neptune are confirmed. John Maddox 